\title{
Cognitive Behaviour Therapy to Treat a Case of Conversion Disorder with Mutism in Bangladesh
}

\author{
Umme Habiba Jasmine ${ }^{1 *}$ and Farah Deeba ${ }^{2}$ \\ ${ }^{1}$ Department of Psychology, PhD candidate, University of the Witwatersrand \\ ${ }^{2}$ Department of Clinical Psychology, University of Dhaka
}

Submission: November 08, 2017; Published: November 21, 2017

*Corresponding author:Umme Habiba Jasmine, Department of Psychology, University of the Witwatersrand; Tel: +88 01993443070;

Email: uhjasm@gmail.com

\begin{abstract}
Objective: CBT as a relatively new practice in Bangladesh has not been tested yet with a broad range of patients. The present study evaluates the effectiveness of Cognitive Behavior Therapy (CBT) that has been tailored to the patient's need, for the treatment of a patient diagnosed with conversion disorder primarily characterized by mutism. Intense chest pain and headache were the other complaints. The patient was a 21 -year old female who had problems uttering words for about six months with several other psychological and physical symptoms. The total therapeutic work was conducted shared room in a government hospital where 6 other psychotherapy sessions were being conducted simultaneously because of lack of the lack of available facilities of private rooms for each patient.
\end{abstract}

Method: Assessment of the patient was done using in-depth clinical interview through writing, observation, self-reported questionnaires and thought diary. The principal therapeutic techniques of CBT used were psycho-education, thought challenge, problem-solving, stopping secondary gains, sleep hygiene, activity scheduling, social skills training, and relaxation exercise.

Result: After six and a half months of intervention she became able to talk and to manage other psychological and physical symptoms.

Conclusion: The result indicates CBT to be an effective intervention for conversion disorder with mutism if modified according to the patient's capacities. This study is unique in using writing as a means of assessment as well as ventilation, and in its extensive use of CBT techniques. Also, even though there was no strict privacy and the patient's reactions could be observed by other people sitting around for different purposes, it brought no fewer therapeutic outcomes.

Keywords: CBT; Conversion disorder; Homeopath; Mutism; Psychosomatic

Abbreviations : CBT: Cognitive Behavioral Therapy; BT: Behavior Therapy; PTSD: Post-Traumatic Stress Disorder; SIAS: Social Interaction Anxiety Scale; AS: Anxiety Scale; DS: Depression Scale; NATs: Negative Automatic Thoughts

\section{Introduction}

Being mute is not always selective mutism but it could also be a conversion disorder, currently known as functional neurological symptom disorder, which is a sub-group of somatic symptom and related disorders (DSM-V). In selective mutism, the person is fully capable of speaking but fails to speak in certain situations though they are expected to speak [1] whereas in conversion disorder it appears as aphonia, an organic condition of inability to produce voice [2].

Although treating conversion disorders by psychoanalytic and behavioral methods were emphasized [3] in recent years with growing interest in using Cognitive Behavioral Therapy (CBT) in clinics worldwide, the efficacy of the method is found considerable $[4,5]$. CBT has been mentioned as an efficacious treatment for the range of the conditions loosely grouped under the somatoform disorders [6], currently known as Somatic Symptom and Related Disorders. Randomized controlled clinical trials found that conversion disorder can be successfully treated with CBT which included the modification of catastrophic cognitions and inappropriate behaviors $[4,7,8]$. A study conducted in Bangladesh showed CBT to be effective in treating general conversion disorder [9] as well.

Therefore, CBT addressing a case of conversion disorder with mutism was considered worth reporting for three reasons: firstly, no literature has been found of treating conversion disorder with mutism with CBT in Bangladesh; secondly, the identification of the case implies that there are existence of such cases in Bangladesh, and thirdly, limited attention has been found worldwide on conversion disorder with mutism which is a 
rare and complex condition. In current literature, this condition apparently seems to be unsuitable for standard CBT procedure, which inspired the authors to assess the effectiveness of CBT with a case representing the symptoms. Moreover, the practice of CBT is relatively new in Bangladesh. It has not been properly tested yet with abroad range of patients. As much as we are using it keeping the theory intact and making the procedure more suitable and tailored for the patients, the investigation is also required so that the practice of CBT gets verified with its ground in anon-western cultural context like Bangladesh and a culture-sensitive version of CBT can be developed if necessary. Limited techniques of Behavior Therapy (BT) and CBT have been successfully applied to treat PTSD (Post-Traumatic Stress Disorder) induced conversion mutism in two case studies $[10,11]$ of partial mutism whereas this current case had a total mutism. Wald, Taylor \& Scamvougeras [11] also recommended examining the effectiveness of combining operant behavioral strategies with CBT, which has been accumulated in this study.

\section{Method}

\section{Sample}

Ms. A, a 21-year-old unmarried female, was an undergraduate student. She was the youngest child of a middle-class family and was reared up by both parents along with her two brothers. Being the youngest and only daughter, she was overprotected by her family members. Different physical illness and episodes of painful rheumatic fever since childhood made her 'center of attention' of the family. All members were extremely supportive whenever she showed any symptoms of physical illness. She developed a strong belief of unconditional acceptance by the family members (shared core belief: I am always loved). She was emotionally very dependent on the eldest brother. Being the youngest, she was to follow everyone else's decision which made her non-assertive and non-expressive since childhood. She was 19 when the family members, especially the eldest brother and father, started disapproving her romantic relationship and attempted to break the relationship by imposing corporal and emotional punishment, and by withdrawing affection. It became a great threat to her. The continuous conflict between Ms. A and her sister-in-law made her always feel anxious, insecure and sad. These events made her confused and fearful, and as a result,her belief of unconditional acceptance by family members was disapproved. These events created fear and helplessness in her mind. She could not protest and lost the courage of expressing feelings.

During that time, there was uncertainty in her study because of some complexity in the academic registration process. These issues ledher to develop some core beliefs like "I am helpless", Nobody understands me", "My future is dark", and some dysfunctional assumptions like "If I protest the injustice happening to me, then I will become alone", "If I point out my sister-in-law's faults, then the eldest brother will be unhappy", "I should tolerate everything to get my eldest brother's affection".
She somehow adapted to such condition but things became unbearable when she was sent to the village forcefully, was locked in a room, and was informed that her boyfriend was going to marry another girl that same day. She could not do anything to stop it or protest about it but screamed continuously and shouted for a long time. She then stopped talking to everyone out of sadness and after a day she found that she was unable to make any sound. Then her other physical symptoms developed. Her eldest brother was a homeopath practitioner who prescribed her homeopath medicine from the very beginning and the symptoms did not reduce. After being unable to talk for six months, which was thought to be malingering by her family members, she was taken to a hospital for her physical symptoms and the medical officer referred her to psychiatry department where she wasdiagnosed with conversion disorder, prescribedpsychiatric medication, and was referred to clinical psychology servicefor CBT.

\section{Assessment}

The exclusive mode of assessment of this case was 'writing' which is atypical to standard CBT. Since the patient was mute she replied to all questions in writing. Clinical in-depth interview, observation, thought diary, sleep diary, Beck hopelessness scale BHS [12] adapted to Bangladeshi culture (20-item two-point scale with KR-20 coefficients ranging from 0.82 to 0.93 and test-retest reliability 0.66); Bengali translated Social Interaction Anxiety Scale (SIAS) [13] a 20 item scale with construct validity 0.86 , cut off score for probable social phobia to be 36 with sensitivity of 0.93 and specificity of 0.60 , with positive predictive value of 0.84 and a negative predictive value of 0.78); Anxiety Scale (AS) [14] developed in Bangladeshi culture (36-item scale five-point Likert-type scale whose cut-off score is 47.5 with high internal consistency, Cronbach alpha $=0.95$, split-half reliability 0.92 , test-retest reliability 0.70 ; with content validity, criterionrelated validity and construct validity); Depression Scale (DS) [15] developed in Bangladeshi culture (30-item five-point Likert-type scale whose cut-off score is 93 with concurrent and construct validity to be 0.716 , split-half reliability 0.76 and testretest reliability 0.599 ); and subjective problem severity ratings were used for assessment.

The patient's baseline problems were assessed in six domains: cognitive, emotional, behavioral, physiological, sociocultural and motivational. The significant findings are as follows:

a) Cognitive- I am guilty; I will not be able to speak anymore; I am valueless and insignificant; I will become alone; nobody believes my words; my future is uncertain.

b) Behavioral-Avoidance of social interaction, reassurance seeking, lack of self-care, avoidance of any real=life issue.

c) Affective- Frustration, intolerance to sympathy, panic, and fear of death, decreased confidence, and feeling confined, feeling anxious and low patience. 
d) Physiological- chest pain, severe headache, smothering, throat pain, loss of appetite, burning sensation, agitation, pounding heart, recurrent sickness, parenthesis, hot and discomfort in chest, tiredness, feeling choked and sickness, insomnia.

e) Socio-environmental- lack of assertion, disharmony with sister-in-law, family members' shock caused by the patient's problems, isolation.

f) Motivational- inattention and disinterest to work, not eager to think deeply.

The patient developed lots of Negative Automatic Thoughts (NATs) ("I will never be able to talk", "I am a tree of sorrow, "I can never take myself back to my previous condition", "Nobody believes me", "I am a person with misfortune", "I will become alone", "All my achievements have gone into vain") which increased her negative feelings and behavior. After the onset of her problem, her whole family became too soft to her, and all the beating, scolding and oblique references were stopped. Besides, the patient had some secondary gains such as receiving extra attention, importance and care, written expression facility for her needs, and keeping away the sister-in-law from her. She also got rid of the burden of household works which became unbearable before the problem onset. She again became the focus of attention of the family. She started communicating through writing. These secondary gains, NATs, dysfunctional thoughts and maladaptive behaviors and the symptoms itself helped in maintaining her problems.

\section{Intervention}

Procedure: The patient's consent was taken before starting assessment. Problem assessment, intervention, and follow-up consisted of 23 sessions including both individual and family psychotherapy sessions. The sessions were designed and devised differently from conventional CBT structure due to her mutism and the physical symptoms. Each session consisted of weekly 90 to 100 minutes instead of standard 50 minutes from the beginning as the patient was completely mute and she had to express her words through writing. It was difficult to conduct each session at a stretch because she could be tired and sick due to her physical symptoms. The psychological conflicts and cognitive biases were identified with guided discovery during the in-depth interview. Sometimes, the patient used to bring a written description of different incidences and current situation. Agenda were set collaboratively at the beginning of each session and the whole session used to progress following the agenda. Pretest assessment included 12 sessions. However, psychometric assessment tools were not administered at every session since patient's expression through writing made assessment process to be too slow. Then formulation was shared in 13th session and the intervention was started from 14 th session.

After the assessment, long-term treatment goal (Developing skills to functionally deal with real life situation) and some short- term treatment goals (To be able to talk; to be assertive; to reduce anxiety related somatic symptoms) were set collaboratively with the patient. From the 15 th session, which was the pick time when the patient had an internal urge of expressing her feelings, the patient's expression through writing in the therapeutic session was stopped and was encouraged to express things verbally. The therapist utilized this urge to help her utter some words for the first time in psychotherapy. Besides, after taking the patient's consent, the therapist discussed the procedure of stopping secondary gains within a family session as part of managing environmental contingency. After 19 sessions, the patient's problems were found significantly reduced by both subjective and objective ratings, and she was gradually prepared for termination. Finally, the case was terminated at the 22nd session and was sent for one-monthfollow-up. The CBT model and applied therapeutic techniques were reviewed at the 22nd session, and it made the patient realizeher own contribution in recovery. This empowered her. The post-test assessment was done at the23rd session.

Intervention Techniques: A number of CBT and BT techniques were used to achieve treatment goals: Psychoeducation about the development of symptoms and prevalence of conversion disorder and related issues was given in 4 th session. Through psycho-education, the patient reported of realizing her problems, becoming normalized, and feeling optimistic about recovery. The Formulationwas shared in the13th session. The patient became very emotional and she was crying intensively while formulation was shared. She accepted the formulation without any objection. Secondary gain was withdrawn after consultation with the patient in 14th session after formulation sharing, which helped the patient to keep trying to make sounds and express needs verbally. It also helped her to overcome other physical, affective and motivational problems rapidly. Upon her consent, her two brothers and two cousins were invited to a family therapy session as she was living with them. Case formulation was also shared with them and they were trained in controlling secondary gains. They were also educated about the negative effects of corporal punishment and were encouraged to stop it.

The patient had a great difficulty in solving personal problems and making decisions. So, she suffered a lot with confusion and this sometimes led her to work impulsively. Problem- solving techniques [16] were discussed with her to overcome this problem. Thought Challenge Technique, developed by Beck [16], was used to challenge the dysfunctional assumptions and negative automatic thoughts (NATs) as she had both of them extensively. Pie-chart was used in the17th session to reduce her guilt feelings by determining relative responsibility [16] in her presented problems for which she made herself responsible. She also went through assertiveness training from 18th session both individually and in group social skill training. It helped the patient to express her opinion, feelings, and rights [17] to reduce interpersonal difficulties with family and friends and it 
was designed for future conflict resolution. Weekly activity diary reflected that she did not keep any pleasurable activity time for herself.

So, a list of pleasurable and purposeful activity plan was made from the patient's past repertoire including shopping, eating, watching TV, chatting, which brought relief from low mood and loss of anhedonia [18]. Then, a graded list of activity was formed in treatment session and weekly activity schedule was done. It also helped in reducing her indecisiveness and motivational problems. Progressive muscular relaxation [19], Imagery and breathing relaxation were applied both individually and in the group as the patient had some physical symptoms due to anxiety and stress. Since Ms. A had insomnia she was taught Sleep hygiene [20] to improve her sleep pattern and to promote good sleep while it is regarded as a behavioral insomnia strategy [21]. In the end, some cognitive and behavioral works were specially designed for relapse prevention. The key to relapse prevention was for the person to continue using the cognitive and behavioral skills learned in treatment, and to recognize the early signs of relapse in order to take steps to prevent.

\section{Result and Discussion}

Progress was evident in both objective and subjective ratings. The initial and the final scores obtained on AS, DS, and BHS are given in Table 1. Measures were used on regular basis with an exception of SIAS. SIAS was administered in the 14th session as the intervention focused on improving her social skills and assertiveness then. The scores show noticeable improvement in anxiety, depression, hopelessness and social interaction anxiety at the end of the intervention. Treatment goals were achieved within 22 sessions. The following table presents the treatment goals and the level of achievements throughout the treatment sessions: Subjective ratings of overall well-being were taken by 0 to 100 scales throughout the therapeutic sessions where 100 meant quite very good state and 0 meant quite a bad state. The patient's improvement by subjective ratings is presented in the following Table 2. The patient and her family members were inclined to homeopath medication. So, she did not take psychiatric medication at all. Rather she took homeopath medication named Kaliphos 6 xs, and Conium Maculatum 30 though on an irregular basis. One limitation of the present study is to rule out the effect of homeopathy medication in her recovery. However, the effectiveness of the prescribed homeopath medication on conversion disorder with mutismhas not been established by any previous study. A study of Seyedaghanoor [22] shows Ignatia Amaramedicine to be effective in curing conversion disorder, which she was not prescribed. Moreover, Ms. A was irregular in taking homeopathy medicine. Another limitation of this study is the difficulty to rule out spontaneous remission for overcoming conversion disorder with mutism. However, Ms. A spent six months with mutism, and somatic and other symptoms without any improvement before starting psychotherapy. If her problems were to remit spontaneously it is likely to start before she started psychotherapy. All these issues and the subjective and objective ratings provide evidence in favor of CBT to be effective for treating conversion disorder which is supported by the studies of Rothbaum and Foa [10], Speed and Mooney [23], and Wald, Taylor and Scamvougeras [11] though they have used limited techniques of CBT (Figure 1).

Table 1: Initial scores of the different scales and their interpretations.

\begin{tabular}{|c|c|c|c|c|}
\hline Name of scales & Initial Score & Interpretation of Score & Score at 21st session & Interpretation of score \\
\hline Anxiety Scale (AS) & 98 & Profound anxiety & 43 & Minimal anxiety \\
\hline $\begin{array}{c}\text { Depression Scale (DS) } \\
\text { Beck Hopelessness Scale } \\
\text { (BHS) }\end{array}$ & 120 & Moderate depression & 72 & Minimal depression \\
\hline $\begin{array}{c}\text { Social Interaction Anxiety } \\
\text { Scale (SIAS) }\end{array}$ & 43 & $\begin{array}{c}\text { Very much social } \\
\text { interaction anxiety }\end{array}$ & 12 & $\begin{array}{c}\text { Slightly social interaction } \\
\text { anxiety }\end{array}$ \\
\hline
\end{tabular}

Table 2: Level of achievement of treatment goals (100\% indicates full achievement and $0 \%$ indicates no achievement at all).

\begin{tabular}{|c|c|c|c|}
\hline Treatment goals & $\mathbf{1 6}^{\text {th }}$ session & $\mathbf{2 0}^{\text {th }}$ session & $\mathbf{2 2}^{\text {nd }} \mathbf{s e s s i o n ~}^{\text {Reducing somatic symptoms }}$ \\
\hline Curing mutism & $70 \%$ & $95 \%$ & $100 \%$ \\
\hline Being assertive & $80 \%$ & 1005 & $100 \%$ \\
\hline $\begin{array}{c}\text { Skillfully deal with real-life } \\
\text { situations }\end{array}$ & $10 \%$ & $80 \%$ & $100 \%$ \\
\hline
\end{tabular}




\section{Psychology and Behavioral Science International Journal}

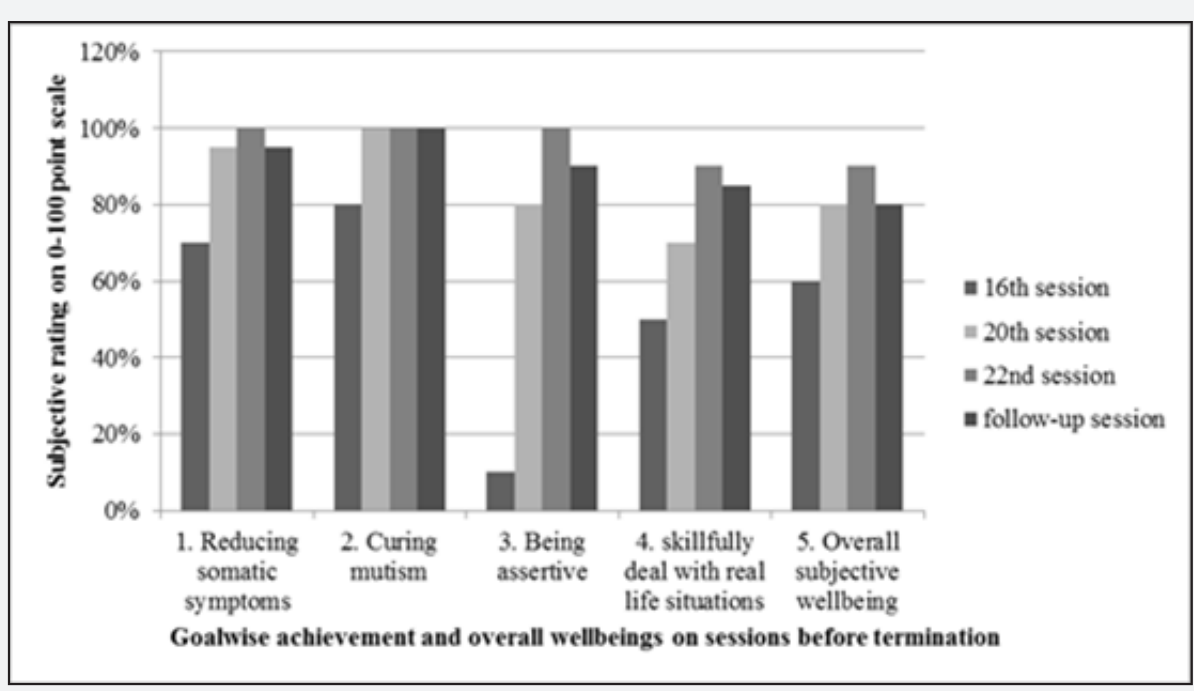

Figure 1: Change in subjective ratings of overall wellbeing throughout the sessions.

\section{Clinical Implication of the Study}

The clinical case reports are the fundamental units to develop scientific investigation in treatment world [24]. In the perspective of the present growing state of the behavioral medicine in Bangladesh, such case studies are important and will contribute to enhance the growth of the scientific profile of the profession. Through the current single-case study, it is concluded that writing as a mode of communication could be an effective strategy in delivering standard CBT for mutism. Also that, expressive writing with adult educated cases having verbalization problem might be beneficial in reducing psychological symptoms. This method assisted the patient to express her memories, feelings and thoughts easily. Usage of writing helped her overcoming primary symptoms, and experience less pain in throat and chest similar to the study findings found with women cases with chronic pelvic pain [25]. Rather than conducting the therapy in a private room required for CBT, the sessions were conducted in a big room where 6 other sessions were being conducted simultaneously by other psychologists with their individual patients. The reason behind is the lack of available facilities of private rooms for each patient. This is how CBT was conducted in a different setting here but brought no fewer outcomes. Even though there were no strict privacy and the patient's reactions could be observed by other people sitting around for different purposes, it could not restrain therapeutic outcome. The possible factors worked behind might be good rapport between the psychotherapist and the patient, the patient's high motivation to overcome her problems, and patient's low expectations about therapeutic environment in a government hospital setting. However, extensive research only can establish these understandings with confidence.

Funding: This research did not receive any specific grant from funding agencies in the public, commercial, or not-forprofit sectors.
Disclosure: The authors report no proprietary or commercial interest in any product mentioned or concept discussed in this article.

\section{References}

1. Adelman L (2007) Don't call me shy: Preparing shy children for a lifetime of social success. Texas Langmarc Publishing.

2. Owens C, Dein S (2006) Conversion disorder: the modern hysteria. Advances in Psychiatric Treatment 12(2): 152-157.

3. Thorpe LU, Keegan DL, Veeman GA (1985) Conversion mutism: case report and discussion. The Canadian Journal of Psychiatry 30 (1): 7173.

4. Kroenke K (2007) Efficacy of treatment for somatoform disorders: A review of randomized controlled trials. Psychosomatic Medicine 69(9): 881-888.

5. Tazaki M, Landlaw K (2006) Behavioural mechanisms and cognitivebehavioural interventions of somatoform disorders. International Review of Psychiatry 18(1): 67-73.

6. Sumathipala A (2007) What is the evidence for the efficacy of treatments for somatoform disorders? A critical review of previous intervention studies. Psychosomatic Medicine 69(9): 889-900.

7. Goldstein LH, Deale AC, Mitchell O'Malley, SJ Toone, BK Mellers (2004) An evaluation of cognitive behavioral therapy as a treatment for dissociative seizures: A pilot study. Cognitive and Behavioral Neurology 17 (1): 41-49.

8. Kroenke K, Swindle R (2000) Cognitive Behavioral Therapy for Somatization and Symptom Syndromes: A critical review of controlled clinical trials. Psychotherapy and Psychosomatics 69(4): 205-215.

9. Tanjin T (2007) Efficacy of Psychological Management of Conversion Disorder. An unpublished M. Phil. Thesis submitted to the Department of Clinical Psychology Bangladesh, India.

10. Rothbaum BA, Foa EB (1991) Exposure treatment of PTSD concomitant with conversion mutism: a case study. Behavior Therapy 22(3): 449456.

11. Wald J, Taylor S, Scamvougeras A (2004) Cognitive behavioral and neuropsychiatric treatment of post-traumatic conversion disorder: a case study. Cognitive Behaviour Therapy 33 (1): 12-20. 
12. Beck AT, Steer RA (1988) Manual for the Beck Hopelessness Scale. Psychological Corp Texas, USA.

13. Mattick RP, Clarke JC (1998) Development and validation of measures of social phobia scrutiny fear and social interaction anxiety. Behaviour Research and Therapy 36: 455-470.

14. Deeba F, Begum R (2004) Development of an anxiety scale for Bangladeshi population. Psychological Studies 14: 39-54.

15. Uddin MZ, Rahman MM (2005) Development of a scale of depression for use in Bangladesh. Bangladesh Psychological Studies 15.

16. Beck JS (1995) Cognitive therapy: Basics and beyond. J Psychother Pract Res 6(1): 71-80.

17. Alberti RE, Emmons ML (1974) Your Perfect Right: A guide to assertive behaviour. San Luis Obispo California.

18. Blackburn IM, Davidson K (1997) Cognitive therapy for depression and anxiety. Cambridge, UK.
19. Jacobson E (1938) Progressive relaxation. Chicago, USA.

20. Hauri PJ (1993) Consulting about insomnia: A method and some preliminary data. Sleep 16(4): 340-350.

21. Lacks P (1987) Behavioral treatment for persistent insomnia. New York, USA.

22. Seyedaghanoor S (2013) Effective homeopathic cure of patient with somatoform disorder. Journal of Homeopathy Ayurvedic Medicine $2: 120$.

23. Speed J, Mooney G (1997) Rehabilitation of conversion disorders: an operant approach. Neuro Rehabilitation 8: 175-181.

24. Janicek M (1999) Clinical case reporting in evidence based medicine. Oxford Butterworth Heinemann.

25. Norman SA, Lumley MA, Dooley JA (2004) For whom does it work? Moderators of the effects of written emotional disclosure in a randomized trial among women with chronic pelvic pain. Psychosom Med 66(2): 174-183.

\section{Your next submission with Juniper Publishers will reach you the below assets}

Commons Attribution 4.0 License

DOI: 10.19080/PBSIJ.2017.07.555716 